\title{
Derivation of the Turbulent Time Scales and Velocity Variances from LES Spectral Data: Application in a Lagrangian Stochastic Dispersion Model
}

\author{
S. Maldaner ${ }^{1}$, G.A. Degrazia ${ }^{*},{ }^{1}$, U. Rizza $^{2}$, S.B.A. Rolim ${ }^{3}$, O.C. Acevedo ${ }^{1}$, E.P. Marques Filho ${ }^{4}$, \\ L.P. Moor ${ }^{1}$ and D.R. Roberti ${ }^{1}$ \\ ${ }^{1}$ Universidade Federal de Santa Maria, Departamento de Física, Santa Maria, Brazil \\ ${ }^{2}$ Istituto di Scienzedell'Atmosfera e del Clima, CNR, Lecce, Italy \\ ${ }^{3}$ Programa de Pós-Graduação em Sensoriamento Remoto, Universidade Federal do Rio Grande do Sul, Porto Alegre, \\ RS, Brazil \\ ${ }^{4}$ Instituto de Geociencias da Universidade Federal do Rio de Janeiro (IGEO-UFRJ), Rio de Janeiro, Brazil
}

\begin{abstract}
Turbulent time scales and velocity variances for a convective boundary layer are derived from large eddy simulation spectral data. Spectral peak frequencies obtained from LES data are used directly in expressions that allow establishing such times scales and velocity variances. These turbulent parameters were compared with those provided by experimental turbulence data. The comparison employing a stochastic dispersion model and observed concentration data shows that both parameterizations reproduce adequately the contaminant dispersion process in a convective boundary layer.
\end{abstract}

Keywords: Convective boundary layer, Lagrangian dispersion model, LES spectral data, Local velocity decorrelation time scale, velocity variances.

\section{INTRODUCTION}

The determination of characteristic time scales and velocity variances associated to geophysical turbulent phenomena is of fundamental importance in contaminant dispersion models. Particularly, such quantities are employed to parameterize the Lagrangian stochastic dispersion models [1].

Concerning the homogeneous turbulence there is an exact definition for the Lagrangian integral time scales which is represented by

$T_{L i}=\int_{0}^{\infty} \rho_{L}(\tau) d \tau$

where $i=u, v, w$ are the velocity components, $\rho_{L i}$ is the dimensionless form of the Lagrangian autocorrelation function and $T_{L i}$ is a measure of the longest time during which, on the average, a fluid particle persists in a motion in a given direction [2]. However, for an inhomogeneous turbulence in a planetary boundary layer (PBL), $T_{L i}$ becomes a local velocity decorrelation time scale and can be expressed as [3],

$T_{i}=\frac{\beta_{i} F_{i}(0)}{4}$

*Address correspondence to this author at the Universidade Federal de Santa Maria, Departamento de Física, Santa Maria, Brazil;

Tel: +555532208616; E-mail: gervasio.degrazia@pesquisador.cnpq.br where $\beta_{i}=\frac{\sqrt{\pi} U}{4 \sigma_{i}}$ is defined as the ratio of the Lagrangian to the Eulerian decorrelation time scale [4], $\mathrm{U}$ is the mean wind speed, $\sigma_{i}$ is the turbulent velocity standard deviation, and $F_{i}(0)$ is the spectrum of turbulent energy normalized by the velocity variance and calculated at the frequency $n \rightarrow 0$. Such time scale, as given by Eq. (2), describes a local memory effect associated with the eddy patterns in a PBL.

Following Degrazia et al. [3] the convective turbulence spectrum in the PBL can be expressed as:

$$
\frac{n S_{i}}{w_{*}^{2}}=\frac{1.06 c_{i} f\left(\psi_{\varepsilon} z / z_{i}\right)^{2 / 3}}{\left[\left(f_{m}\right)_{i}\right]^{5 / 3}\left\{1+1.5 f /\left[\left(f_{m}\right)_{i}\right]\right\}^{5 / 3}}
$$

where $c_{i}=\alpha_{i} \alpha_{u}(2 \pi k)^{-2 / 3}$ and $\alpha_{u}=0.5 \pm 0.05$ and $\alpha_{i}=1,4 / 3,4 / 3$ for $u, v$ and $w$ components, respectively [5, 6], $f=n z / U$ is reduced frequency, $z$ is the height above of the ground, $z_{i}$ is the convective boundary layer height, $\psi_{\varepsilon}=\varepsilon z_{i} / w_{*}^{3}$ is the adimensional dissipation rate function, $w_{*}$ is the convective velocity scale, $\varepsilon$ is the buoyant rate of turbulent kinetic energy dissipation and $\left(f_{m}\right)_{i}$ is the normalized frequency of peak spectral. By analytically integrating the Eq. (3) over the whole frequency domain one can obtain the following turbulent velocity variance: 
$\sigma_{i}^{2}=\frac{1.06 c_{i} \psi_{\varepsilon}^{2 / 3} w_{*}^{2}\left(z / z_{i}\right)^{2 / 3}}{(f m)_{i}^{2 / 3}}$

The velocity variances indicate the turbulence intensity and consequently its ability to disperse the contaminants

Therefore, normalizing the energy spectrum (Eq. 3) by the velocity variance (Eq. 4), yields

$$
F_{i}(0)=\frac{z}{U\left(f_{m}\right)_{i}}
$$

Substituting Eq. (5) into Eq. (2) results the following equation:

$T_{i}=\frac{1}{4} \frac{\beta_{i} z}{U\left(f_{m}\right)_{i}}$

Both formulations for $T_{L i}$ and $\sigma_{i}$ (Eqs. (4) and (6)), are described in terms of the reduced frequency of the energy spectral peak. Such fundamental frequency provides time and length scale associated to the energy-containing eddies of the turbulent flow. As a consequence, such parameters can be used in dispersion models. Therefore, $(f m)_{i}$ is important for investigations of turbulent transport in the PBL. Generally, expressions for $(f m)_{i}$ are almost all obtained from fitting expressions to experimental data. However, experimental data of this fundamental parameter connected to the turbulence energy spectra are fairly difficult to obtain, particularly, if such observations are extended to the whole depth of the PBL. Not many of such observations are, therefore, available, and for this reason large-eddy simulation (LES) models constitute quite a useful tool to that purpose.

The aim of the present study is to obtain new algebraic formulations for the local velocity decorrelation time scales and turbulent velocity variances in a convective boundary layer (CBL). These relations are obtained from Eqs. (4) and (6), in which $(\mathrm{fm})_{i}$ is derived from a simulation employing a LES model. An additional aim is to apply a well known Lagrangian stochastic dispersion model and concentration data obtained from Prairie Grass classical short-range dispersion experiment to compare these new expressions for $\sigma_{i}$ and $T_{L i}$ with the formulations given by Eqs. (4) and (6), in which $(\mathrm{fm})_{i}$ is obtained from field experimental data.

\section{VERTICAL PEAK WAVELENGTH FROM LARGE EDDY SIMULATION}

Large eddy simulation (LES) models represent a powerful computational methodology to describe the physical properties of the planetary boundary layer $[7,8]$. In LES, the large eddies (energy-containing eddies) of the turbulent field are explicitly resolved and the contribution of the smaller eddies (sub-filter scales) is parameterized. Generally, the basic equations in the LES model are the incompressible Navier-Stokes equations. The resolved turbulent field parameters (velocity components, potential temperature and pressure) are calculated by the application of a low-pass spatial filter which presents a particular dimension, known as the turbulent resolution length scale.

In this study, a numerical simulation of the convective boundary layer was performed employing the LES code of Moeng [8] with Sullivan et al. [9] subfilter parameterization. The filtered Navier Stokes equations for the resolved turbulent flow time dependent variables in the LES of Moeng [8] are written as:

$$
\begin{aligned}
& \frac{\partial \bar{u}}{\partial t}=\overline{\bar{v} \bar{\zeta}_{z}}-\overline{w \overline{\zeta_{y}}}+f \bar{v}-\frac{\partial P^{*}}{\partial x}-\frac{\partial<P>}{\partial x}-\frac{\partial \tau_{x x}}{\partial x}-\frac{\partial \tau_{x y}}{\partial y}-\frac{\partial \tau_{x z}}{\partial z} \\
& \frac{\partial \bar{v}}{\partial t}=\overline{\bar{w} \overline{\zeta_{x}}}-\overline{\bar{u} \overline{\zeta_{z}}}-f \bar{u}-\frac{\partial P^{*}}{\partial x}-\frac{\partial<P>}{\partial x}-\frac{\partial \tau_{x y}}{\partial x}-\frac{\partial \tau_{y y}}{\partial y}-\frac{\partial \tau_{y z}}{\partial z} \\
& \frac{\partial \bar{w}}{\partial t}=\overline{\bar{u} \bar{\zeta}_{y}}-\overline{\bar{v} \bar{\zeta}_{x}}+g \frac{\bar{\theta}}{\theta_{0}}-\frac{\partial P^{*}}{\partial z}-\frac{\partial \tau_{x z}}{\partial x}-\frac{\partial \tau_{y z}}{\partial y}-\frac{\partial \tau_{z z}}{\partial z} \\
& -\left\langle\overline{\bar{u} \overline{\zeta_{x}}}-\overline{\bar{v} \bar{\zeta}_{x}}+g \frac{\bar{\theta}}{\theta_{0}}-\frac{\partial P^{*}}{\partial z}-\frac{\partial \tau_{x z}}{\partial x}-\frac{\partial \tau_{y z}}{\partial y}-\frac{\partial \tau_{z z}}{\partial z}\right\rangle
\end{aligned}
$$

where the brackets represent horizontal means, $\zeta$ are the vorticity components, $\mathrm{g}$ is the gravitational acceleration, $\mathrm{f}$ is the Coriolis parameter, $\bar{\theta}$ is the virtual potential temperature, $\mathrm{P}$ is the pressure and $\tau$ are the subfilter Reynolds stress. On the other hand, the virtual potential temperature is calculated from the following equation:

$$
\frac{\partial \bar{\theta}}{\partial t}=-\overline{-\bar{u} \frac{\partial \bar{\theta}}{\partial x}}-\overline{\bar{v}} \overline{\frac{\partial \bar{\theta}}{\partial y}}-\overline{\bar{w} \frac{\partial \bar{\theta}}{\partial z}}-\frac{\partial \tau_{\theta x}}{\partial x}-\frac{\partial \tau_{\theta y}}{\partial y}-\frac{\partial \tau_{\theta z}}{\partial z}
$$

where $\tau_{\theta}=v_{\theta} \frac{\partial \theta}{\partial x}$, being $v_{\theta}$ the eddy diffusivity for heat

The subfilter Reynolds stress in Eqs. (7) are parameterized in the LES model employing the Sullivan well known parameterization (9) which is described by

$$
\tau_{i j}=-2 v_{t} \delta S_{i j}-2 v_{T}\left\langle S_{i j}\right\rangle
$$

where $S_{i j}$ is the resolved flow rate of strain tensor, $v_{t}$ and $v_{T}$ are respectively the fluctuating and mean field eddyviscosities and $\delta$ is the isotropy factor.

The numerical experiments utilized in this study were accomplished with constant values of the kinematic turbulent heat flux and of the geostrophic wind. A $(5,5,2) \mathrm{Km}$ box domain with variable points in each direction were used in five simulations. From each LES numerical simulation was obtained a spatial spectrum. These spatial spectra were calculated according to Moeng and Wyngaard [10] using a bidimensional fast fourier transform of the tridimensional fluctuations of the turbulent velocity components. Therefore, the spectral peak wavelengths were estimated from these spatial spectra generated of the five LES numerical simulations. The spectra generated from these simulations are described in detail in Marques Filho [11]. Therefore, from the numerical spectral data generated from the LES simulations were derived the following expressions for the vertical and horizontal peak wavelengths: 
$\left(\lambda_{m}\right)_{w}=1.3 z_{i}\left[1-\exp \left(-4.8 z / z_{i}\right)-0.005 \exp \left(4.8 z / z_{i}\right)\right]$

$\left(\lambda_{m}\right)_{u, v}=(1.29 \pm 0.05) z_{i}$

\section{DERIVATION OF VELOCITY VARIANCES OBTAINED OF LES AND OBSERVATIONAL DATA}

Defining $\left(f_{m}\right)_{w}=z /\left(\lambda_{m}\right)_{w}$ in Eq. (4) and employing $\Psi_{\varepsilon}=0.65$ and $c_{w}=0.4$ [12], yields the following relation for $\sigma_{w}^{2}$

$\sigma_{w}^{2}=0.37 w_{*}^{2}\left[1-\exp \left(-4.8 z / z_{i}\right)-0.005 \exp \left(4.8 z / z_{i}\right)\right]^{2 / 3}$

On the other hand, accomplishing an identical development and utilizing the following observational vertical wavelength at the spectral peak proposed by [13]

$$
\left(\lambda_{m}\right)_{w}=1.8 z_{i}\left[1-\exp \left(-4 z / z_{i}\right)-0.0003 \exp \left(8 z / z_{i}\right)\right]
$$

it is possible to obtain a different relation for the vertical velocity variance:

$$
\sigma_{w}^{2}=0.44 w_{*}^{2}\left[1-\exp \left(-4 z / z_{i}\right)-0.0003 \exp \left(8 z / z_{i}\right)\right]^{2 / 3}
$$

Fig. (1) shows observational data [13] and a comparison between the vertical velocity variances given by the Eqs. $(12,14)$. The normalized vertical velocity variance, described by the Eqs. $(12,14)$, presents a parabolic shape with values between 0.3 and 0.4 in height range $0.2<z / z_{i}<0.6$ (middle of the convective boundary layer). It is important to note that both formulations for $\sigma_{w}^{2}$ reproduce well the observational data in the shallow region close to the ground.

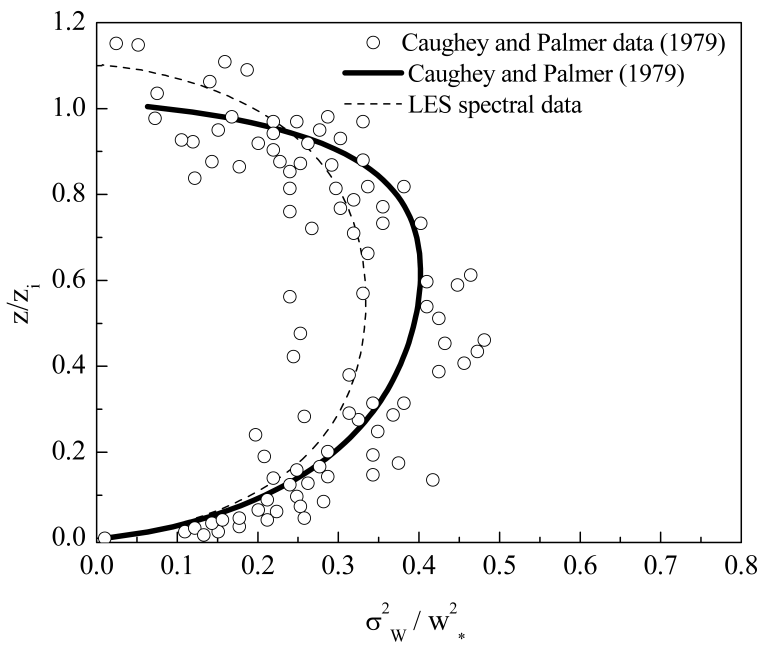

Fig. (1). Comparison between the vertical velocity variances (Eqs. $12,14)$.

To obtain the lateral and longitudinal velocity variances we employ Eq. (4) considering $\Psi_{\varepsilon}=0.65, c_{v}=0.4$, $c_{u}=0.27$ with $\left(f_{m}\right)_{u, v}=z /\left(\lambda_{m}\right)_{u, v} \quad$ The lateral and longitudinal wavelength at the spectral peak, $\left(\lambda_{m}\right)_{u, v}$, provided by the LES simulation, is given by the Eq. (11). The substitution of these values in Eq. (4) yields

$\sigma_{u}^{2}=0.24 w_{*}^{2}, \sigma_{v}^{2}=0.37 w_{*}^{2}$

On the other hand, according to [14] the observed lateral and longitudinal wavelength at the spectral peak is described by $[14,15]\left(\lambda_{m}\right)_{u, v}=1.5 Z_{i}$. Therefore, the observed horizontal velocity variances can be written as:

$\sigma_{u}^{2}=0.28 w_{*}^{2}, \sigma_{v}^{2}=0.41 w_{*}^{2}$

The values for $\sigma_{u}^{2}$ and $\sigma_{v}^{2}$ calculated from LES spectral data are in agreement with the horizontal diffusion data in a water-tank experiment $[16,17]$. Furthermore, the differences between the numerical constants in Eqs. (15) and (16) are not physically relevant.

\section{DERIVATION OF TURBULENT TIME SCALES OBTAINED OF LES AND OBSERVATIONAL DATA}

A formulation for the local vertical velocity decorrelation time scale from LES spectral data can be obtained using Eqs. (10) and (12) into Eq. (6), yielding:

$$
T_{L_{w}}=0.23 \frac{z_{i}}{w_{*}}\left[1-\exp \left(-4.8 z / z_{i}\right)-0.005 \exp \left(4.8 z / z_{i}\right)\right]^{2 / 3}
$$

On the other hand, using Eqs. (13) and (14) into Eq. (6) results the following observational time scale:

$$
T_{L_{w}}=0.29 \frac{z_{i}}{w_{*}}\left[1-\exp \left(-4 z / z_{i}\right)-0.0003 \exp \left(4 z / z_{i}\right)\right]^{2 / 3}
$$

Fig. (2) presents the vertical profile of the local time scales obtained from Eqs. (17) and (18). It can be seen that both formulations present an identical form. There is not a remarkable difference between the two profiles.

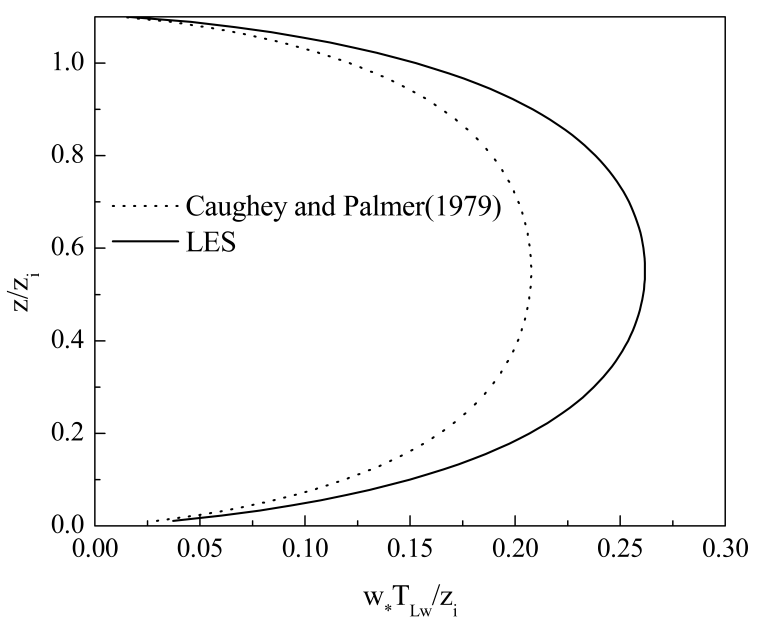

Fig. (2). Profiles of the local times scales (Eqs. 17, 18).

The lateral and longitudinal time scales from LES spectral data can be obtained employing Eqs. (11) and (15) into Eq. (6) resulting: 


$$
T_{L u}=0.29 \frac{z_{i}}{w_{*}} \text { and } T_{L v}=0.23 \frac{z_{i}}{w_{*}}
$$

On the other hand, using $\left(\lambda_{m}\right)_{u, v}=1.5 Z_{i}$ from observational data $[14,15]$ and Eq. (16) into Eq. (6), the following times scales can be written as:

$$
T_{L u}=0.58 \frac{z_{i}}{w_{*}} \text { and } T_{L v}=0.40 \frac{z_{i}}{w_{*}}
$$

The formulations for the horizontal turbulent time scales as given by the Eqs. (19) and (20) are in agreement with the expressions suggested by $[16,18]$.

\section{EMPLOYMENT OF TIME SCALES AND VELOCITY VARIANCES OBTAINED OF LES SPECTRAL DATA IN A LAGRANGIAN DISPERSION MODEL}

The following section aims to use a Lagrangian stochastic dispersion model employing turbulent parameters given by the Eqs. $(12,14,15,16,17,18,19,20)$ to simulate the ground-level cross wind integrated concentrations of contaminants released from a low continuous source in a CBL. Lagrangian Stochastic particle models are numerical tools for the study of the atmospheric turbulent diffusion process. In such models, the fluid particle movements are generated by random velocities and the displacement of a particle is a Markov process. These models are based on Langevin equation. In this work, the Lagrangian Particle Model LAMBDA is employed to simulate the dispersion of passive contaminants. LAMBDA is a Lagrangian Stochastic Dispersion Model (LSDM) based on a three-dimensional form of the Langevin equation for the random velocity $[19,20]$. Thus, the velocity and displacement of each fluid particle, in each time step, is obtained by numerical integration of the following equations [19, 21]:

$$
d u_{i}(t)=a_{i}(\vec{x}, \vec{u}, t) d t+b_{i j}(\vec{x}, \vec{u}, t) d W_{j}
$$

and

$$
d x_{i}=u_{i} d t
$$

where $i, j=1,2,3, d W_{j}$ is the incremental Wiener process, $a_{i}(\vec{x}, \vec{u}, t)$ is a deterministic term, $b_{i j}(\vec{x}, \vec{u}, t) d W_{j}$ is a stochastic term, $\vec{u}$ is the Lagrangian velocity vector and $\vec{x}$ is the displacement vector $[20,21]$. The term $b_{i j}(\vec{x}, \vec{u}, t)$ can be derived from the Kolmogorov theory of local isotropy in the inertial subrange. On the other hand, the deterministic term is provided from the stationary Fokker-Planck equation. In the present study, the Lambda model employs a Gaussian probability density function (PDF) on the horizontal plane and a Gram-Charlier PDF, truncated to the fourth order, in vertical. Wind speed profiles has been parameterized following the similarity theory of Monin-Obukhov and OML model [20]:

$$
\begin{aligned}
& U(z)=\frac{u_{*}}{k}\left[\ln \left[\frac{z}{z_{0}}\right]-\Psi_{m}\left[\frac{z}{L}\right]+\Psi_{m}\left[\frac{z_{0}}{L}\right]\right] \text { if } z<z_{b} \\
& U(z)=U\left(z_{b}\right) \text { if } z>z_{b}
\end{aligned}
$$

where $z_{b}=\min [|L|, 0.1 h], k=0.4$ is the Von Karman constant, $u_{*}$ is the friction velocity, $z_{0}$ is the roughness length, $\mathrm{L}$ is the Monin-Obukhov length and $\Psi_{m}$ is a stability function given by [20]:

$$
\Psi_{m}=2 \ln \left[\frac{1+A}{2}\right]+\ln \left[\frac{1+A^{2}}{2}\right]-2 \tan ^{-1} A+\frac{\pi}{2}
$$

and

$A=\left[1-16 \frac{z}{L}\right]^{1 / 4}$

In this study, we simulate the Prairie Grass diffusion experiment. The Prairie Grass diffusion experiments are described in detail in [20] and [22].

In this atmospheric dispersion experiment, the pollutant $\left(\mathrm{SO}_{2}\right)$ was emitted at a height of $0.5 \mathrm{~m}$ and it was measured by samplers at a height of $1.5 \mathrm{~m}$ in five downwind distances $(50,100,200,400,800 \mathrm{~m})$. In LAMBDA simulations the horizontal domain was determined according to sampler distances and the vertical domain was set equal to the observed mixing height. The time step was maintained constant and it was obtained according to the value of Lagrangian decorrelation time scale $\left(\Delta t=T_{L i} / c\right)$, where $T_{L i}$ must be the smaller value between $T_{L u}, T_{L v}$ and $T_{L w}$ and $\mathrm{c}$ is an empirical coefficient set equal to 10 . One hundred particles were released in each time step during 1000 time steps.

The simulation results employing the turbulent local time scales and velocity variances, obtained from LES spectral data (Eqs. 12, 15, 17, 19), are presented in Fig. (3). Fig. (3) presents the scatter diagram between observed and predicted ground-level cross-wind integrated concentrations. Therefore, in order to establish a comparison between LES and observational turbulent parameters we simulate the Prairie Grass contaminant concentration data employing the formulations given by the Eqs. (14, 16, 18 and 20). Fig. (3) shows also this comparison. Table 1 presents the results of the statistical analysis made with observed and simulated values of the ground-level cross-wind integrated concentration. This table presents statistical indices for the simulations using LES spectral and observational turbulent parameters. LAMBDA model, employing the Eqs. $(12,15$, 17, 19) derived from LES spectral data, shows a good performance, with correlation coefficient ( $\mathrm{R}$ ) near to 1 and normalized mean square error (NMSE), fractional standard deviation (FS) and fractional bias (FB) near to zero. Fig. (3) and statistical indices show that the simulation results reproduce well the observed concentration data for both turbulent parameterizations (LES spectral and observational). From this statistical viewpoint we may conclude that the LAMBDA model utilizing the Eqs. $(12,15$, $17,19)$, representing the phenomenon of the passive scalars dispersion, simulates fairly well the concentration observational data in a convective PBL. It is important, at this point, to consider that such results from the present analysis, employing LES data, have a distinct character from those obtained from observational data. The normalized 
frequencies of the spectral peak, used in the studies from [13] and [15], were derived from fitting equations to observational data, while in the present investigation the data are generated from a LES spectral simulation. The importance of this difference lies in the fact that experimental data of the turbulence spectra are quite difficult to obtain, particularly considering that the observation points must span the depth of the CBL. As a consequence, very few turbulent spectral observations are available and for this reason, LES spectral data constitutes quite a useful tool for that purpose. The present work has, therefore, shown that such LES methodology, to investigate complex turbulent statistical properties, is physically adequate and that it provides compatible results when compared with observational methodology. Furthermore, LES methodology allows better spatial representation, not restrained to vertical levels where the observations are accomplished.

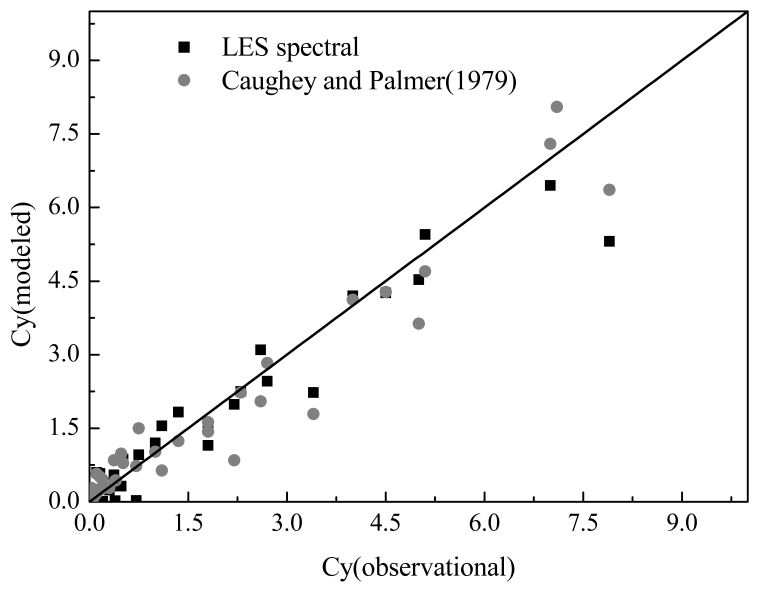

Fig. (3). Scatter diagram between observed and predicted groundlevel cross-wind integrated concentrations $\left(C_{Y}\right)$.

Table 1. Statistical evaluation of the LAMBDA performance.

\begin{tabular}{|l|c|c|c|c|}
\hline \multirow{2}{*}{ Eqs. $(12,15,17,19)$} & NMSE & FS & FB & $\mathrm{R}$ \\
\cline { 2 - 5 } & 0.08 & -0.06 & 0.02 & 0.93 \\
\hline Eqs. $(14,16,18,20)$ & 0.04 & 0.05 & 0.04 & 0.96 \\
\hline
\end{tabular}

\section{CONCLUSION}

Turbulent local time scales and velocity variances are fundamental statistical parameters for passive scalars dispersion modeling. Generally, such quantities are derived from the measured turbulent energy spectra. In this case, the spectral peak wavelengths are employed in formulations that provide the turbulent time scales and velocity variances. However, such observations for the whole vertical region of the CBL are extremely difficult to accomplish. Therefore, in present study, we apply LES simulation data to obtain algebraic formulations for the turbulent local time scales and velocity variances in a CBL. These expressions are derived from Eqs. $(4,6)$ in which the peak frequencies are extracted directly from LES spectral data. The analysis shows that there is a fairly good agreement between the times scales and velocity variances derived from LES data with those provided by turbulence observations in the CBL. Finally, in order to test the times scales and velocity variances derived from LES data we introduce the new turbulent parameterization in a Lagrangian stochastic dispersion model and, employing concentration data from dispersion field experiments, we compare them with formulations obtained from observations accomplished in a CBL. Evaluating the results and related statistical indices it can be seen that the LAMBDA dispersion model reproduces adequately the measurements of experimental concentration with the turbulent time scales and velocity variances calculated from LES spectral data. It can be concluded that good contaminant concentration simulations are determined with the turbulent approach obtained from LES spectral data. This analysis shows that when LES derived Eqs. $(12,15,17,19)$ are utilized in a dispersion model, results are tantamount to those extracted from field observations. The major difference lies in the fact that LES simulations results give more detailed information about of the distinct parameters of the CBL and do not depend on difficult and expensive observations, which present also some degree of uncertainty. The good quality of the results obtained from Lagrangian Dispersion model employing both the LES spectral turbulent time scales and velocity variances or those obtained experimentally shows that either of these parameterizations may be applied with equivalent results.

\section{CONFLICT OF INTEREST}

The authors confirm that this article content has no conflict of interest.

\section{ACKNOWLEDGEMENTS}

The authors thank CAPES (Coordenação de Aperfeiçoamento de Pessoal de Nível Superior), CNPq (Conselho Nacional de Desenvolvimento Científico e Tecnológico) and FAPERGS (Fundação de Apoio a Pesquisa do Estado do Rio Grande do Sul) for partial financial support of this work.

\section{REFERENCES}

[1] Rodean HC. Stochastic lagrangian models of turbulent diffusion. Johnson DR, Ed. Meteorological Monographs. Am Meteorol Soc, Boston 1996; 26(48).

[2] Hinze JO. Turbulence, McGraw-Hill Book Company, New York 1975.

[3] Degrazia GA, Anfossi D, Carvalho J, Mangia C, Tirabassi T, Campos Velho H. Turbulence parameterization for PBL dispersion models in all stability conditions. Atmos Environ 2000; 34: 357583 .

[4] Wandel CF, Kofoed-Hansen O. On the eulerian-lagrangian transform in the statistical theory of turbulence. J Geophys Res 1962; 76: 3089-93.

[5] Champagne FH, Friehe JC, La Rue JC, Wyngaard JC. Flux measurements, flux estimation techniques, and fine-scale turbulence measurements in the unstable surface layer over land. J Atmos Sci 1977; 34: 515-30.

[6] Sorbjan Z. Structure of the atmospheric boundary layer. Prentice Hall, New Jersey 1989, pp.317.

[7] Deardorff JW. The use of subgrid transport equations in a threedimensional model of atmospheric turbulence. In: American Society of Mechanical Engineers, Applied Mechanics and Fluids Engineering Conference, Atlanta 1973.

[8] Moeng CH. A large-eddy-simulation model for the study of planetary boundary-layer turbulence. J Atmos Sci 1984; 41: 205262. 
[9] Sullivan PP, McWilliams JC, Moeng C-H. A subgrid-scale model for large-eddy simulation of planetary boundary-layer flows. Boundary-Layer Meteorol 1994; 71: 247-76.

[10] Moeng CH, Wyngaard JC. Spectral analysis of large-eddy simulations of the convective boundary layer. J Atmos Sci 1988; 45: 3573-87.

[11] Marques Filho EP. Investigação da camada limite planetária convectiva com modelo LES aplicado à dispersão de poluentes [online]. São Paulo: Instituto de Astronomia, Geofísica e Ciências Atmosféricas, Universidade de São Paulo, 2004. Doctorate Dissertation in Meteorology. [Access on 2013-09-24]. Available at: http://www.teses.usp.br/teses/disponiveis/14/14133/tde-20082004$134629 /$

[12] Højstrup J. Velocity spectra in the unstable planetary boundary layer. J Atmosph Sci 1982; 39: 2239-48.

[13] Caughey SJ, Palmer SG. Some aspects of turbulence structure through the depth of the convective boundary layer. Quart J Roy Meteor Soc 1979; 105: 811-27.

[14] Caughey SJ. Observed characteristics of the atmospheric boundary layer. In: Nieuwstadt FTM, van Dop H, Eds. Atmospheric turbulence and air pollution modelling. Reidel Dordrecht 1982; pp. 107-58.

[15] Kaimal JC, Wyngaard JC, Haugen DA, et al. Turbulence structure in the convective boundary layer. J Atmosph Sci 1976; 33(11): $2152-69$
[16] Dosio A, Vilà-Guerau de Arellano J, Holtslag AA, Builtjes PJ. Dispersion of a passive tracer in buoyancy-and shear-driven boundary layers. J App Meteorol 2003; 42: 1116-30.

[17] Degrazia GA, Rizza U, Puhales FS, et al. Employing Taylor and Heisenberg subfilter viscosities to simulate turbulent statistics in LES models. Physica A: Statist Mechan Applicat 2012; 391: 102031.

[18] Luhar AK. The influence of vertical wind direction shear on dispersion in the convective boundary layer, and its incorporation in coastal fumigation models. Boundary-Layer Meteorol 2002; 102: $1-38$.

[19] Thomson DJ. Random walk modelling of diffusion in inhomogeneous turbulence. Quart J Royal Meteorological Soc 1984; 110: 1107-20.

[20] Carvalho JD, Degrazia GA, Anfossi D, De Campos CRJ, Roberti DR, Kerr AS. Lagrangian stochastic dispersion modelling for the simulation of the release of contaminants from tall and low sources. Meteorol Zeitschrift 2002; 11: 89-97.

[21] Ferrero E. Lagrangian particle models (theory). In: Zanetti P. Ed, Air Quality modeling: theories, methodologies, computational techniques and available databases and software. Enviro Comp Inst 2005; pp. 93-161.

[22] Barad M. Project prairie grass: A field program in diffusion. Geophysical Research Paper II, Report AFCRC-TR-58-235(II), Air Force Cambridge Research Center, USA, 1958.

(C) Maldaner et al.; Licensee Bentham Open.

This is an open access article licensed under the terms of the Creative Commons Attribution Non-Commercial License (http://creativecommons.org/licenses/by-nc/3.0/) which permits unrestricted, non-commercial use, distribution and reproduction in any medium, provided the work is properly cited. 